\title{
Magnetic resonance imaging findings compared with histological findings of the labrum in hip osteoarthritis
}

\author{
Shiho Kanezaki • Asako Yamamoto • Shigeru Nakamura • \\ Marie Osawa • Takashi Matsushita
}

Received: 31 July 2014 /Revised: 24 December 2014 / Accepted: 5 January 2015 /Published online: 24 January 2015

(C) ISS 2015

\section{Introduction}

Patients with disorders such as acetabular dysplasia or femoroacetabular impingement are at risk of developing hip osteoarthritis. Assessment of the cartilage and labrum in the hip joint based on magnetic resonance imaging (MRI) has been challenging because of the low signal-to-noise ratio (SNR) due to its deep location, ball and socket structure, and small volume of those structures compared with the whole joint size. To achieve better imaging assessment, direct MR arthrography (d-MRA) [1] and other techniques such as T2* mapping [2], T2 mapping [3], T1rho [4], and delayed gadolinium-enhanced MRI (dGEMRIC) [5] have been developed along with the increasing use of high-field MRI. In patients with no apparent osteoarthritic changes such as joint space narrowing or osteophyte and subchondral cyst formation on radiographs, these techniques can detect early cartilage or labral damage [6].

A recently developed semiquantitative MRI-based scoring system for hip osteoarthritis [7] includes evaluation of the labrum, and its application as a potential therapeutic monitoring tool is anticipated. The labrum shows pathological changes such as macroscopic hypertrophy and histological degeneration in hip osteoarthritis, but the pathological background is not well understood when evaluated by MRI. Kubo et al. [8] compared radial MRI findings with histological changes of

S. Kanezaki $(\bowtie) \cdot$ S. Nakamura $\cdot$ T. Matsushita Department of Orthopaedic Surgery, Teikyo University School of Medicine, 2-11-1 Kaga, Itabashi-ku, Tokyo 173-8605, Japan e-mail: starlovedear122@yahoo.co.jp

A. Yamamoto $\cdot$ M. Osawa

Department of Radiology, Teikyo University School of Medicine, Itabashi-ku, Tokyo, Japan the labrum in 11 hips with osteoarthritis using 1.5-T MRI and found that fibrous separation and mucoid deposition occurred in the labrum with a "diffuse high signal" or "obscure" pattern. However, to the best of our knowledge, no studies have demonstrated a correlation between MRI findings and histological evidence of the severity of degeneration of the labrum.

We hypothesized that radially reconstructed images of the acetabular labrum acquired by 3-T MRI can depict degenerative changes of the labrum. In this study, we sought to determine the correlation between MRI and histological findings of the labrum in osteoarthritic hips.

\section{Materials and methods}

Patients

Institutional review board approval was obtained for this study. Total hip arthroplasty (THA) was performed for 44 osteoarthritic hips in 43 patients (4 males and 39 females) between the 3rd week of April 2012 and the 3rd week of February 2013. The diagnosis of osteoarthritis of the hip was based on the American College of Rheumatology criteria for hip osteoarthritis [9]. Patients diagnosed as having rheumatoid arthritis according to the diagnostic criteria of the American Rheumatoid Association [10] or idiopathic osteonecrosis of the femoral head according to the Japanese Ministry of Health, Labour, and Welfare [11] were excluded. Of the 43 patients, 28 provided informed consent to participate in the study. One patient was subsequently excluded because of insufficient imaging quality due to severe motion artifacts. Thus, a total of 28 hips (18 right and 10 left) in 27 patients ( 1 male and 26 females) were included in the study. The mean age of patients at 
the time of surgery was 67 years (range, $44-79$ years). The Tönnis grade of radiographic arthritis [12] was grade 2 (moderate loss of joint space) in 4 hips and grade 3 (severe localized loss of joint space) in 24 hips. Twenty-one of these 28 hips had acetabular dysplasia with a center edge angle $<20^{\circ}$ on anteroposterior radiographs.

\section{MRI protocol}

MRI examination was performed preoperatively, and the average duration between MRI examination and THA was 29 days (range, 5-68 days). All images were acquired using a 3-T imaging unit (Signa HDxt, GE Healthcare, Milwaukee, WI) with an eight-channel phased array cardiac coil. Threedimensional (3D) T2*-weighted images were acquired using the multiecho recalled gradient echo (MERGE) sequence with the following settings: repetition time/echo time, 35.7/ $17.7 \mathrm{~ms}$; slice thickness, $1.0 \mathrm{~mm}$; field of view, $24 \mathrm{~cm}$; matrix size, $288 \times 224$; flip angle, $5^{\circ}$; number of excitations, 1 ; bandwidth, $41.7 \mathrm{kHz}$; scan time, $10 \min 59 \mathrm{~s}$.

Double-oblique images corrected for acetabular anteversion and acetabular inclination angle were reconstructed. On the axial reconstructed images (Fig. 1a), the anterior and posterior edges of the acetabulum were identified in the slice that best demonstrated the midpoint of the femoral head, and the images perpendicular to the line passing through the two points was reconstructed (oblique coronal plane; Fig. 1b). On the oblique coronal images of the midpoint of the femoral head, the superior and inferior edges of the acetabulum were identified, and the plane passing through the two points was reconstructed (double-oblique sagittal plane; Fig. 1c). The double-oblique sagittal plane included the entire circumference of the acetabular margin. Radially reconstructed sections passing through the midpoint of the acetabulum and crossing vertically to the acetabular margin made it possible to observe the whole labrum continuously [8].

The acetabulum was divided into five zones based on the clock position: the anterior, anterosuperior, posterosuperior, posterior, and inferior parts were designated as zones $1-5$, respectively. With the image of the continuous radially reconstructed sections, four planes passing through the center of each zone (excluding zone 5) via the midpoint and one plane passing through the border between zones 2 and 3 (zone 2/3) were evaluated. All cases were reviewed on a clinical workstation (Ziostation2, Ziosoft, Mita, Tokyo).

The slice was regarded as "unidentified" if the labrum could not be identified. The evaluation criteria for the radially reconstructed slices of the labrum were as follows: 1 , size; 2 , shape; 3 , signal intensity. To determine the size, the labral length at the femoral head side was measured and classified into three groups: large $(>10 \mathrm{~mm})$, medium $(4-10 \mathrm{~mm})$, and small $(<4 \mathrm{~mm})$. In assessment of the shape, the labrum was regarded as triangular if both borders were smooth with a
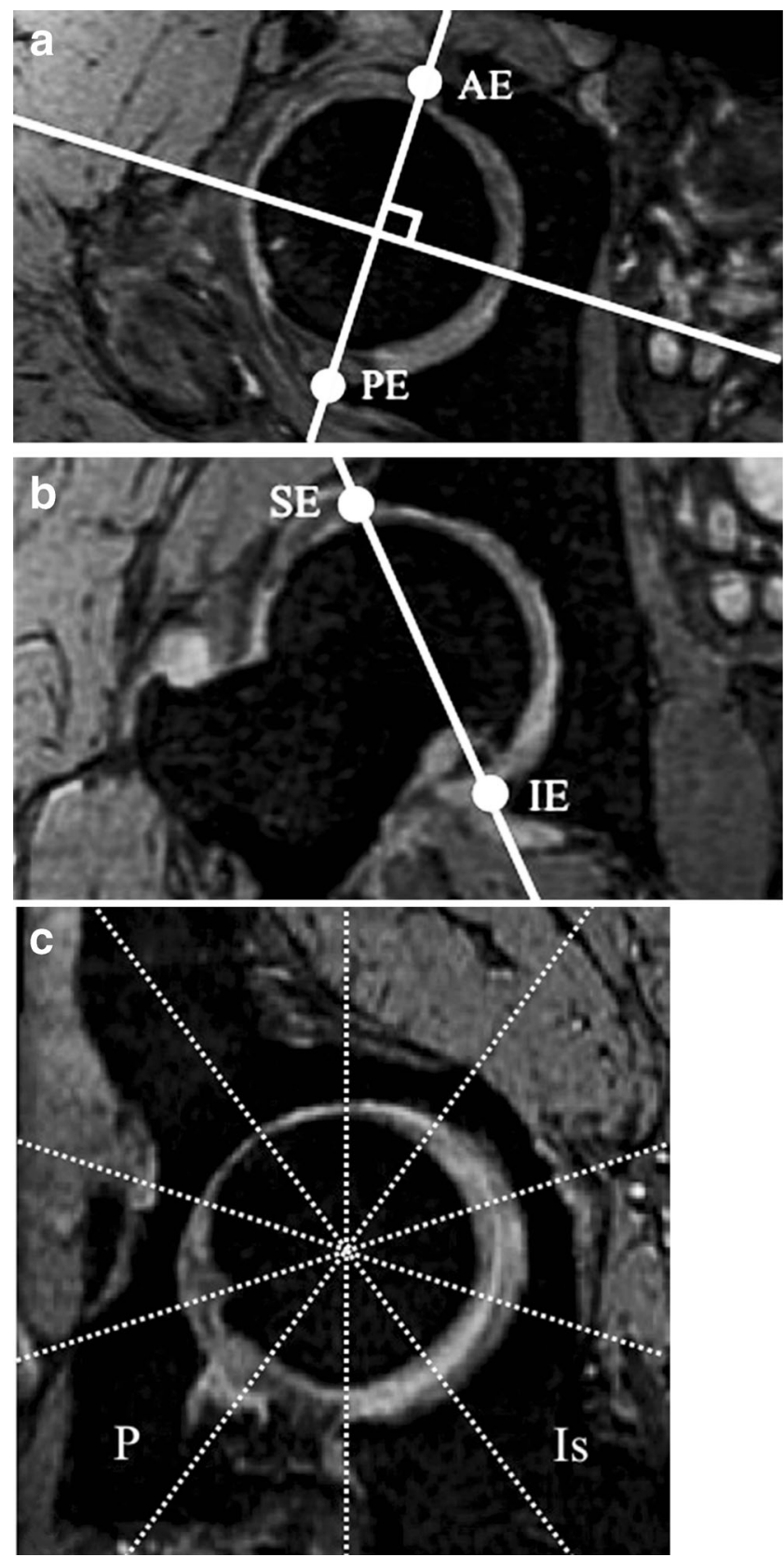

Fig. 1 Reconstruction of double-oblique sagittal plane magnetic resonance images. The double-oblique sagittal plane was reconstructed as follows: in the axial reconstructed image (a), the anterior and posterior edges of the acetabulum were identified in the slice that best demonstrated the midpoint of the femoral head, and the images perpendicular to the line passing through the two points was reconstructed (oblique coronal plane). In the oblique coronal image (b) passing through the midpoint of the femoral head, the superior and inferior edges of the acetabulum were identified, and the image passing through the two points was reconstructed (double-oblique sagittal plane). In the double-oblique sagittal image (c), five radial dotted lines depict the directions of the slices that were used for evaluations. AE, anterior edge; $\mathrm{PE}$, posterior edge; SE, superior edge; IE, inferior edge; Is, ischium; $\mathrm{P}$, pubis

sharp edge, and the others were regarded as non-triangular. Detachment of the labrum was recorded when the continuity 
between the labrum and acetabulum was completely lost with interruption by a fluid signal.

Signal intensity was measured manually using the regionof-interest (ROI) measurement tool [13-15], with the labrum margin being enclosed in the polygonal ROI in the five radially reconstructed slices (Fig. 2). A circular ROI was set in the ipsilateral rectus femoris muscle tendon on an axial reconstructed slice at the level of the lesser trochanter, and the average signal intensities of the labrum and rectus femoris muscle tendon were recorded. The signal intensity ratio (SIR) for each patient was calculated using the following formula: average signal intensity of the labrum/average signal intensity of the rectus femoris muscle tendon. One radiologist (AY, 9 years of experience) reconstructed the slice and evaluated the size and shape. Two radiologists (AY, MO; 4 years of experience) had reached a consensus on the way to put ROIs on the labrum and the femoral rectus tendon in the reconstructed images before calculations were performed. The two readers independently evaluated signal intensities in the ROIs and calculated the SIR in a blinded fashion.

\section{Surgical technique}

Surgeries were performed by the third author (SN) using a posterior approach with the patient in the lateral decubitus position. A minimal incision was made to the posterior capsule to avoid injury to the labrum, and the entire labrum was inspected after resecting the femoral head. The acetabulum was divided into five zones based on the clock position of the acetabulum as described above (Fig. 3). Zone 5 was not inspected because it was occupied by the transverse ligament. The first author (SK) recorded the presence of major detachment of the labrum. Markings were made at the cranial portion

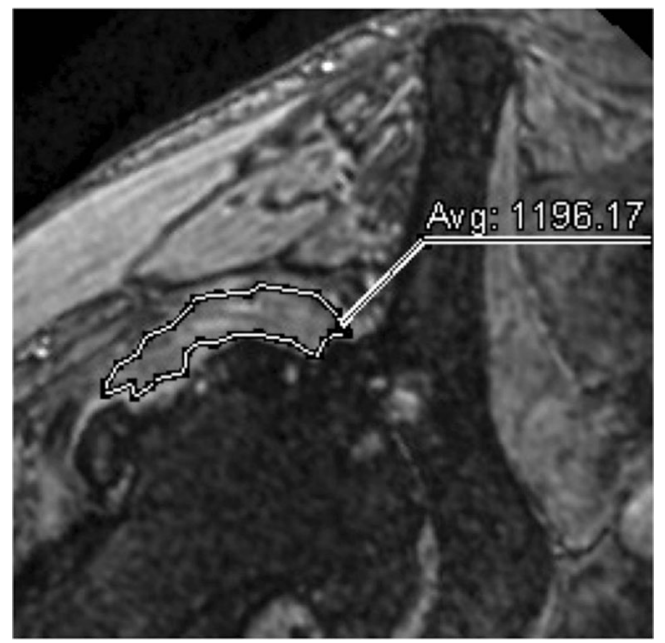

Fig. 2 Placement of the polygonal ROI on the labrum. The polygonal ROI enclosing the labrum margin is shown in the radially reconstructed MRI image. Avg, average signal intensity in the ROI

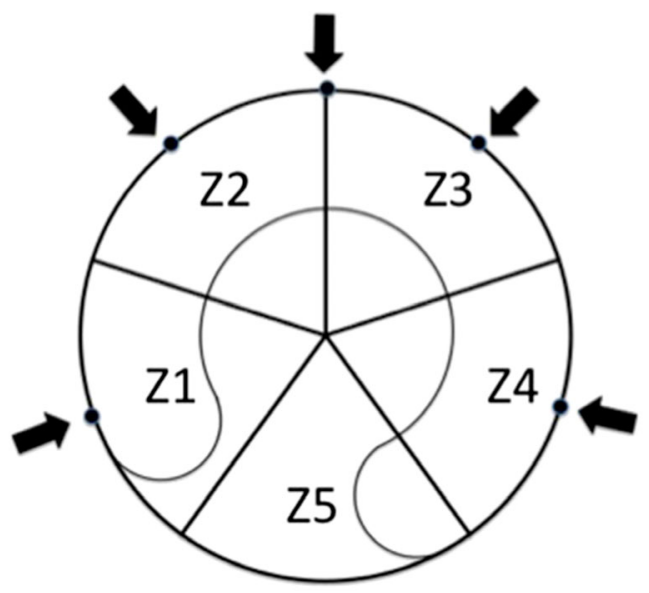

Fig. 3 The five zones and clock positions of the acetabulum. The acetabulum was divided into five zones: zone 1, anterior part; zone 2, anterosuperior part; zone 3, posterosuperior part; zone 4, posterior part; zone 5 , inferior part. Zone $2 / 3$ was designated as the 12 o'clock position. The five points (arrows) were selected for macro- and microscopic evaluation of the radial sections

of the labrum ( 12 o'clock) and posterior portion ( 9 o'clock for the right hip and 3 o'clock for the left hip) with absorbable 2-0 sutures to prevent confusion regarding labral orientation. Next, the labrum was resected en block from the bony acetabular rim using a scalpel.

\section{Macroscopic assessment}

Gross inspection of the resected labrum was performed immediately, followed by fixation in $5 \%$ formalin. The fixed labrum was placed on a paper with a pre-drawn circle divided into the five zones and oriented with the cranial suture aligned to zone $2 / 3$ and the posterior suture to zone 4 . The centers of zones $1-4$ and zone $2 / 3$ were selected as the five points for gross and macroscopic evaluation of the radial sections. Labral specimens with radial sections of $3 \mathrm{~mm}$ were taken from each of these five points.

The macroscopic evaluation criteria for radial sections pertained to size and shape. To determine the size, the width of the femoral head side from the free edge to the attachment of the acetabular rim was measured and classified as large $(>10 \mathrm{~mm})$, medium $(4-10 \mathrm{~mm})$, or small $(<4 \mathrm{~mm})$. In the assessment of shape, the sections were categorized as triangular or non-triangular as described in the MRI procedure.

\section{Microscopic assessment}

Labrum specimens were embedded in paraffin, sectioned into $3-\mu \mathrm{m}$ slices, and stained with hematoxylin and eosin. The grade of degeneration was histologically categorized into three stages according to the presence of normal fibrocartilage: grade 0 (normal fibrocartilage $\geq 2 / 3$ ), grade 1 (normal fibrocartilage $\geq 1 / 3$ and $<2 / 3$ ), and grade 2 (normal 
fibrocartilage $<1 / 3$ ). The first author (SK) and third author (SN) evaluated the histological findings independently without referring to the MRI findings.

MRI compared with macro- and microscopic findings

If all evaluation items including presence (identified/unidentified), size, shape, and detachment of the labrum were the same between the MRI and macroscopic findings, the results were defined as concordant, and the concordance rate was calculated. Furthermore, correlations were evaluated between the SIR, which was calculated on MRI, and histological grading.

Statistical analysis

Interclass correlation coefficients were used to analyze the intra- and interobserver reliability for SIR on MRI. Intraobserver reliability was analyzed by comparing the first and second evaluations of one musculoskeletal radiologist (AY), and interobserver reliability was analyzed by comparing the findings of two musculoskeletal radiologists (AY and MO). The intra- and interobserver reliability analysis of SIR showed good consistency, with interclass correlation coefficients of 0.740 [95\% confidence interval (CI): 0.652-0.809] and 0.848 (95\% CI: $0.790-0.890)$, respectively.

Weighted kappa was used to analyze the intra- and interobserver reliability for histological grading. Intraobserver reliability was analyzed by comparing the first and second evaluations of the first author (SK), and interobserver reliability was analyzed by comparing the findings of the first author (SK) and third author (SN). The intra- and interobserver reliability analysis of histological grading showed good consistency, with weighted kappas of 0.959 (95 \% CI: 0.920-0.999) and 0.868 (95 \% CI: $0.794-0.941$ ), respectively.

Correlations between SIR and histological grading were evaluated using Spearman's rank correlation coefficient. All statistical analyses were performed with SAS 9.3 (SAS Institute, Cary, NC).

\section{Results}

Of the 140 MRI slices obtained (5 slices for each of the 28 hips), a retractor placed on the labrum in zone 1 resulted in artificial injuries to the labral specimens during surgery for 3 hips, and the MRI slices corresponding to these specimens were therefore excluded from the analysis. The remaining 137 slices were evaluated and compared with the macroscopic findings.

The findings for the presence, size, shape, and detachment of the labrum are shown in Table 1. On MRI, the labrum could
Table $1 \mathrm{MRI} /$ macroscopic findings for the presence, size, shape, and detachment

\begin{tabular}{|c|c|c|c|c|c|}
\hline Hip no. & Zone 1 & Zone 2 & Zone $2 / 3$ & Zone 3 & Zone 4 \\
\hline 1 & $\mathrm{U} / \mathrm{U}$ & $\mathrm{LN} / \mathrm{LN}$ & $\mathrm{LN} / \mathrm{LN}$ & $\mathrm{LN} / \mathrm{LN}$ & $\mathrm{LT} / \mathrm{LT}$ \\
\hline 2 & $\mathrm{U} / \mathrm{U}$ & $M N / M T$ & $\mathrm{LN} / \mathrm{LN}$ & $\mathrm{LN} / \mathrm{LN}$ & MT/MT \\
\hline 3 & $\mathrm{U} / \mathrm{U}$ & $\mathrm{LN} / \mathrm{LN}$ & $\mathrm{MN} / \mathrm{MN}$ & $\mathrm{LN} / \mathrm{LN}$ & $\mathrm{LN} / \mathrm{LN}$ \\
\hline 4 & $M T / M N$ & $L N / L T$ & $\mathrm{LN} / \mathrm{LN}$ & $\mathrm{LT} / \mathrm{LT}$ & $\mathrm{LN} / \mathrm{LN}$ \\
\hline 5 & $M N / S N$ & $\mathrm{LN} / \mathrm{LN}$ & MT/MT & $\mathrm{LT} / \mathrm{LT}$ & $\mathrm{LN} / \mathrm{LN}$ \\
\hline 6 & $L N / L T$ & $\mathrm{LN} / \mathrm{LN}$ & $\mathrm{LN} / \mathrm{LN}$ & $\mathrm{LN} / \mathrm{LN}$ & MT/MT \\
\hline 7 & $\mathrm{MN} / \mathrm{MN}$ & $\mathrm{LN} / \mathrm{LN}$ & $L N / L T$ & $\mathrm{LT} / \mathrm{LT}$ & $\mathrm{LN} / \mathrm{LN}$ \\
\hline 8 & $L T / S T$ & $L T / L N$ & $\mathrm{LN} / \mathrm{LN}$ & $\mathrm{LN} / \mathrm{LN}$ & $\mathrm{LT} / \mathrm{LT}$ \\
\hline 9 & $M T / M N$ & $\mathrm{LN} / \mathrm{LN}$ & $\mathrm{LN} / \mathrm{LN}$ & $\mathrm{LN} / \mathrm{LN}$ & MT/MT \\
\hline 10 & $\mathrm{MN} / \mathrm{MN}$ & $\mathrm{LN} / \mathrm{LN}$ & $\mathrm{LN} / \mathrm{LN}$ & $\mathrm{LN} / \mathrm{LN}$ & $\mathrm{LN} / \mathrm{LN}$ \\
\hline 11 & $L T / M N$ & $U / M N$ & $\mathrm{LN} / \mathrm{LN}$ & $\mathrm{LN} / \mathrm{LN}$ & $\mathrm{LN} / \mathrm{LN}$ \\
\hline 12 & $\mathrm{LN} / \mathrm{LN}$ & $\mathrm{LN} / \mathrm{LN}$ & $\mathrm{LN} / \mathrm{LN}$ & $L N / L T$ & $\mathrm{LT} / \mathrm{LT}$ \\
\hline 13 & $\mathrm{U} / \mathrm{U}$ & $\mathrm{U} / \mathrm{U}$ & $\mathrm{LN} / \mathrm{LN}$ & $\mathrm{LT} / \mathrm{LT}$ & $\mathrm{LN} / \mathrm{LN}$ \\
\hline 14 & $\mathrm{LT} / \mathrm{LT}$ & $\mathrm{LT} / \mathrm{LT}$ & $M T / L T$ & $\mathrm{LT} / \mathrm{LT}$ & MT/MT \\
\hline 15 & $L T / L N$ & $\mathrm{LT} / \mathrm{LT}$ & $\mathrm{LN} / \mathrm{LN}$ & $L T / L N$ & $\mathrm{LT} / \mathrm{LT}$ \\
\hline 16 & $\mathrm{LT} / \mathrm{LT}$ & $\mathrm{LN} / \mathrm{LN}$ & $\mathrm{LN} / \mathrm{LN}$ & $\mathrm{LN} / \mathrm{LN}$ & $\mathrm{LN} / \mathrm{LN}$ \\
\hline 17 & $M T / M N$ & $\mathrm{LN} / \mathrm{LN}$ & $\mathrm{LN} / \mathrm{LN}$ & $\mathrm{LT} / \mathrm{LT}$ & $\mathrm{MT} / \mathrm{MT}$ \\
\hline 18 & MT/MT & $\mathrm{LN} / \mathrm{LN}$ & $M T / L N$ & $\mathrm{LT} / \mathrm{LT}$ & $M T / L T$ \\
\hline 19 & MT/MT & LND/LND & MND/MND & $\mathrm{LN} / \mathrm{LN}$ & MT/MT \\
\hline 20 & $\mathrm{MN} / \mathrm{MN}$ & LND/LND & $\mathrm{LN} / \mathrm{LN}$ & $\mathrm{LN} / \mathrm{LN}$ & MT/MT \\
\hline 21 & - & $\mathrm{LN} / \mathrm{LN}$ & $\mathrm{LT} / \mathrm{LT}$ & $\mathrm{LT} / \mathrm{LT}$ & $M T / M N$ \\
\hline 22 & MT/MT & $M N / M T$ & $U / M N$ & $\mathrm{LN} / \mathrm{LN}$ & $L N / L T$ \\
\hline 23 & $\mathrm{LN} / \mathrm{LN}$ & $\mathrm{LN} / \mathrm{LN}$ & $\mathrm{LN} / \mathrm{LN}$ & $\mathrm{LN} / \mathrm{LN}$ & $\mathrm{LT} / \mathrm{LT}$ \\
\hline 24 & $\mathrm{MT} / \mathrm{MT}$ & $U / L T$ & $\mathrm{LT} / \mathrm{LT}$ & $\mathrm{LT} / \mathrm{LT}$ & $\mathrm{MT} / \mathrm{MT}$ \\
\hline 25 & - & $\mathrm{LN} / \mathrm{LN}$ & $\mathrm{LN} / \mathrm{LN}$ & $\mathrm{LT} / \mathrm{LT}$ & $\mathrm{MT} / \mathrm{MT}$ \\
\hline 26 & $\mathrm{LT} / \mathrm{LT}$ & $L N D / L T D$ & $\mathrm{LN} / \mathrm{LN}$ & $\mathrm{LT} / \mathrm{LT}$ & $\mathrm{LT} / \mathrm{LT}$ \\
\hline 27 & $\mathrm{U} / \mathrm{U}$ & $\mathrm{LN} / \mathrm{LN}$ & $\mathrm{LN} / \mathrm{LN}$ & $\mathrm{LN} / \mathrm{LN}$ & $\mathrm{LN} / \mathrm{LN}$ \\
\hline 28 & - & $\mathrm{LN} / \mathrm{LN}$ & $U / M T$ & $\mathrm{MN} / \mathrm{MN}$ & MT/MT \\
\hline
\end{tabular}

U, unidentified; -, specimens were excluded; L, large; M, medium; S, small; $\mathrm{T}$, triangular; $\mathrm{N}$, non-triangular; $\mathrm{D}$, detachment

Italics: discordance between MRI and macroscopic findings

not be identified in ten slices, while macroscopically the labrum was absent in six zones. The sensitivity, specificity, positive predictive value, and negative predictive value were 97 , 100,100 , and $60 \%$, respectively, for detecting the presence of the labrum. The macroscopic findings and MRI findings in four slices were not concordant with respect to the presence/ absence of the labrum: labra were detected macroscopically in four of the ten unidentifiable slices on MRI. These four slices were located in zone 2 or at 12 o'clock, associated with severe bony destruction.

On MRI, the size of the identified labrum was classed as large in 96 slices, medium in 31, and small in none, while macroscopic size was large in 98 specimens, medium in 31, and small in 2. On MRI, the shape of the labrum was classed as triangular in 51 slices and non-triangular in 76 , while macroscopically it was triangular in 52 specimens and non- 
triangular in 79. The most frequent combination of size and shape was large and non-triangular (61 specimens) (Fig. 4). Labral detachment on MRI (Fig. 5) was observed in four slices, while macroscopic labral detachment was observed in four specimens corresponding to the four slices showing detachment on MRI. Findings in $81 \%(112 / 137)$ of the MRI slices were concordant with the macroscopic findings for all evaluations, including the presence, size, shape, and detachment of labrum.

After excluding the $10 \mathrm{MRI}$ slices with unidentified labra, the mean SIR of the remaining 127 MRI slices was 2.89 (range, 1.06-6.46). Microscopic findings revealed various ratios of normal fibrocartilage and pathological changes including myxoid degeneration, fibrillation, neovascularization, cystic change, and hyaline cartilaginous change (Fig. 6). The
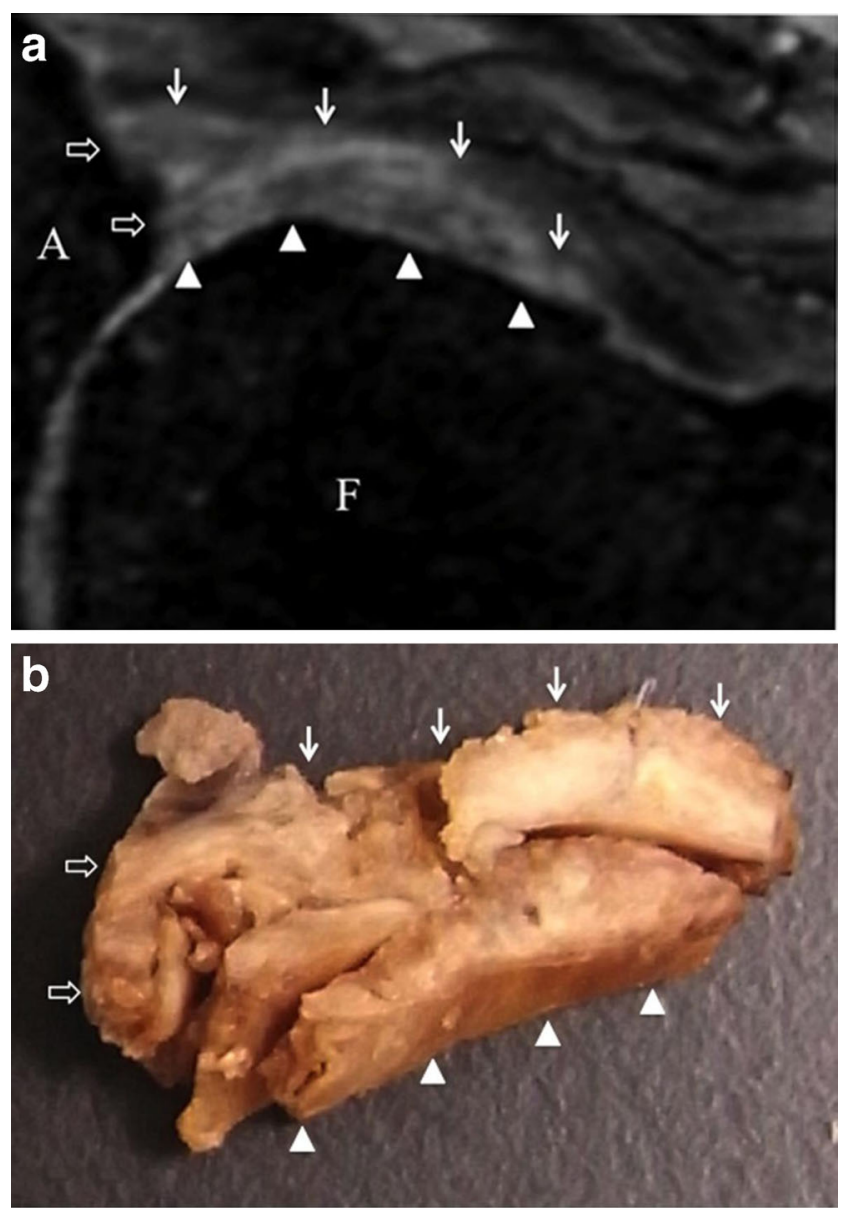

Fig. 4 MRI and macroscopic findings of a large, non-triangular-shaped labrum (case 6; zone 2, SIR, 3.15). a Radially reconstructed MRI showing a labral length of $30 \mathrm{~mm}$ and a non-triangular shape. The arrows are on the articular capsule side of the labrum, the arrowheads are on the femoral head side of the labrum, and the box arrows are at the labral attachment side of the acetabulum. A, acetabulum; F, femoral head. b Macroscopic findings showing a labral length of $28 \mathrm{~mm}$ and a nontriangular shape, which were concordant with MR findings. The arrows are on the articular capsule side of the labrum, the arrowheads are on the femoral head side of the labrum, and box arrows are at the labral attachment side of the acetabulum

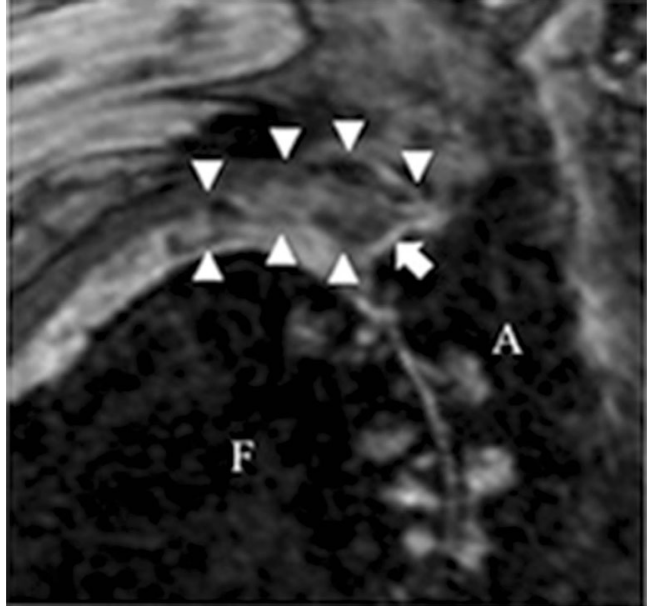

Fig. 5 Detachment of the labrum on MRI. Continuity between the labrum (arrowheads) and the acetabulum was completely lost with interruption by a fluid signal (arrow). A, acetabulum; F, femoral head

histological grading of degeneration was grade 0 in 24 , grade 1 in 54, and grade 2 in 49 specimens. The four specimens with detachments showed myxoid degeneration and cystic change at the attachment side of the acetabular rim. Grade 2 was most frequently found at zone $2(33 \%)$, while grade 0 was most frequently found at zone 4 (63\%). Regarding the relationship between MRI and microscopic findings, a substantial correlation was found between SIR and histological grade (Spearman's rank correlation coefficient, 0.568) (Fig. 7). The mean SIR was 2.21 (range, 1.06-4.45) for grade 0, 2.72 (range, 1.53-5.80) for grade 1, and 3.39 (range, 2.20-6.46) for grade 2. A higher SIR was associated with more advanced degeneration.

\section{Discussion}

The acetabular labrum is a fibrocartilage structure attached to the peripheral rim of the acetabulum that exhibits a triangular shape in the radial section macroscopically. On MRI slices, the normal labrum appears as a low-signal triangular structure attached to the acetabular margin, and abnormalities are usually defined by changes in shape and signal intensity [6]. However, several studies of healthy volunteers showed variations in the MRI appearance of the labrum [16, 17]. Aydingöz et al. [17] studied MRI images of the labrum in both hips of 180 asymptomatic volunteers and found age-related changes in signal intensity. Hodler et al. [18] reported the signal change of the basal labrum in elderly cadavers. They speculated that the signal change could be due to the presence of small fibrovascular bundles or an irregular zone of labral insertion. Although such age-related signal change has been widely recognized today, its pathological basis is still not well known. The opportunity for comparison with macroscopic and microscopic findings is rare in normal or early stages of OA. In the hip 
Fig. 6 Microscopic findings of the labrum. Specimens were stained with hematoxylin and eosin. Some specimens had mainly normal fibrocartilage (a), while others showed pathological findings including myxoid degeneration (arrows) (b), fibrillar fuzzing on the surface of the fibrocartilaginous tissue indicating fibrillation (c), zonal proliferation of capillaries (arrows) indicating neovascularization (d), cystic change (arrows) (e), and a lobular pattern of cartilaginous cells and matrices indicating hyaline cartilaginous change $(\mathrm{f})$. Bar= $100 \mu \mathrm{m}(\mathbf{a}, \mathbf{b}, \mathbf{d}, \mathbf{e}), 200 \mu \mathrm{m}(\mathbf{c})$, and $50 \mu \mathrm{m}$ (f)

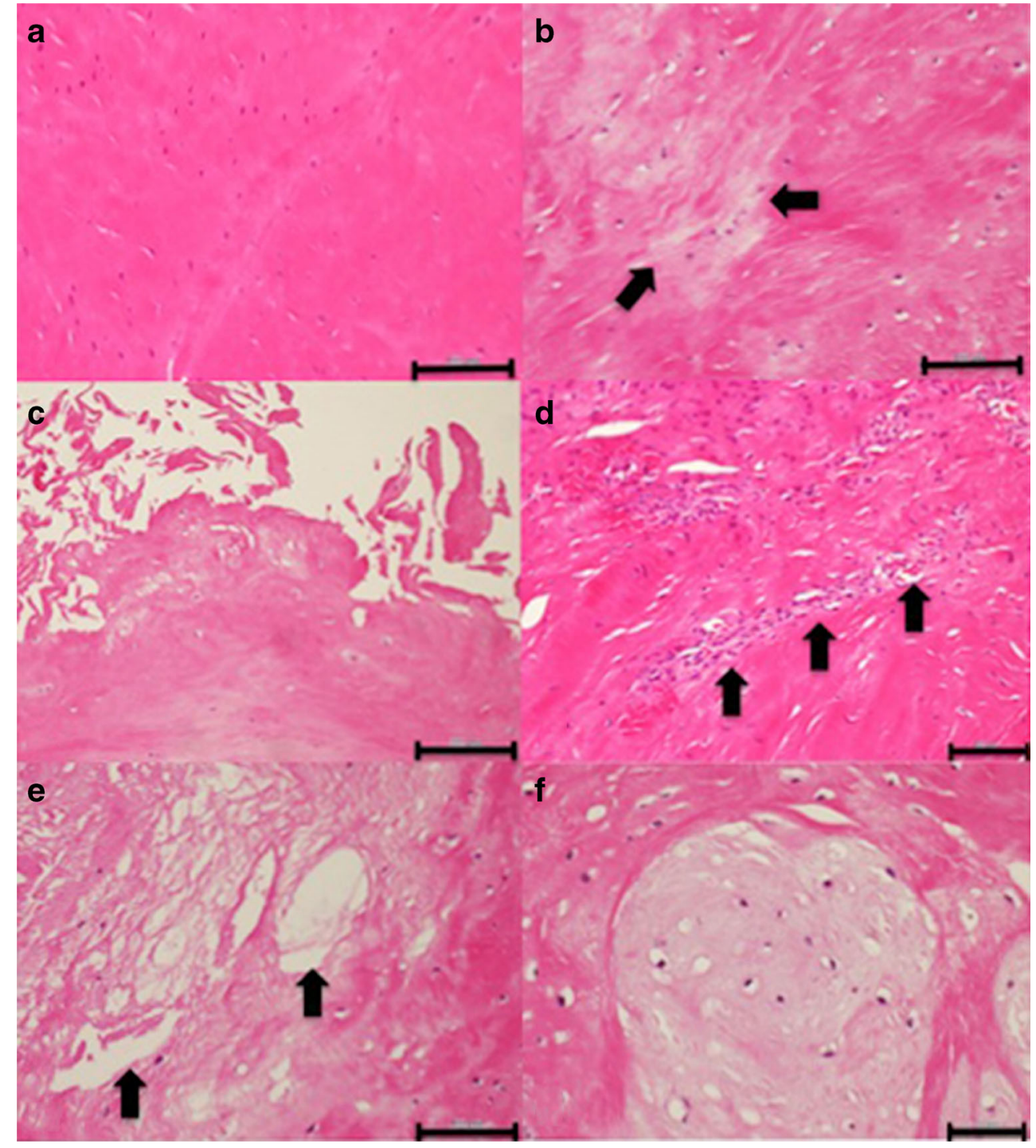

with advanced or end-stage osteoarthritis, the labrum could suffer from not only severe degeneration in the weightbearing area, but also mild degeneration in the non-weightbearing area. Therefore, correlation between radiological and pathological findings was examined in patients from whom the whole labrum could be resected during THA in the present study.

Advances in hip arthroscopy during the past few decades have made possible less invasive surgery for intra-articular abnormalities in disorders such as femoroacetabular impingement $[19,20]$. Intra-articular abnormalities including labral and osteochondral lesions can be assessed with recent MRI techniques before arthroscopic interventions, and many studies have evaluated the correlation between arthroscopic and MRI findings of the labrum in hips with no or early osteoarthritis. d-MRA remains the most commonly used and validated technique for evaluating the labrum but requires a twostage procedure in which a gadolinium-based contrast agent is injected intra-articularly under fluoroscopic guidance followed by MRI within $30 \mathrm{~min}$ of the injection. Although high sensitivity and specificity have been reported for dMRA, intra-articular injection of contrast solution is associated with complications such as contrast media allergy and pyogenic arthritis [21]. On the other hand, some studies have reported the effectiveness of non-contrast MRI for diagnosing labral and chondral abnormalities [22, 23]. In a review article, Gold et al. [6] stated that both high-resolution non-contrast MRI and d-MRI provide reproducible and accurate assessment of hip cartilage and the labrum.

Surgical treatment of hip osteoarthritis includes arthroscopic interventions, acetabular osteotomy, and THA. THA is usually indicated for hips with advanced or end-stage osteoarthritis in elderly patients. Acetabular osteotomy such as rotational acetabular osteotomy (RAO) or peri-acetabular osteotomy (PAO) can be performed for dysplastic hips with early or advanced osteoarthritis [24, 25]. Both techniques were 


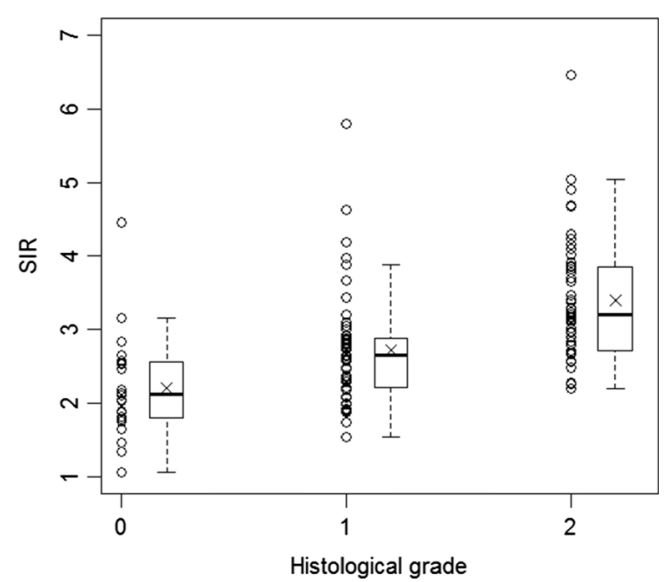

Fig. 7 Correlation between SIR and histological grading. A substantial correlation was found between SIR and histological grading (Spearman's rank correlation coefficient, 0.568). The box-and-whisker plots of SIR in each grade are shown. The box consists of median (central line) and upper and lower quartiles (top and bottom lines, respectively). The whisker ends show the maximum and minimum values within the 1.5 interquartile range from the upper and lower quartiles, respectively. $\mathrm{X}$ shows the mean value

developed for painful dysplastic hips in adolescents and young adults and have demonstrated good long-term outcomes in hips with mild osteoarthritis [26]. In addition, some studies reported that RAO outcomes were also favorable for some hips with advanced osteoarthritis [24, 25]. In these studies, preoperative evaluations were conducted using plain radiographs. Recently, preoperative MRI evaluations have been recommended for patient selection for RAO or PAO [27, 28]. Cunningham et al. [27] evaluated cartilage using d-GEMRIC before PAO in 47 patients and reported that the d-GEMRIC index was an important predictor of osteotomy failure. Their study group included hips with radiographic osteoarthritis of Tönnis grade 2 or grade 3, but the labrum was not evaluated. Kim et al. [29] performed concomitant hip arthroscopy with PAO and suggested that the arthroscopic treatment of labral pathology alters the progression of osteoarthritis. The present results suggest that preoperative evaluation of the labrum by MRI provides useful information for patient selection for PAO or RAO with concomitant arthroscopy.

MRI evaluation of the labrum is also useful for predicting progressive joint space narrowing in mild hip dysplasia [30]. However, few studies have evaluated the correlation between MRI findings and degenerative changes of the labrum. To improve the MRI plane for depicting the acetabular labrum in more detail without associated invasive procedures such as the use of a contrast agent, Kubo et al. [8] developed radialsequence MRI, which displays the entire acetabular circumference. Although they found fibrous separation and mucoid deposition occurred on portions of the labrum where a diffuse high signal or obscure pattern was seen with 1.5-T MRI, they did not evaluate correlations between MRI findings and the degree of histological degeneration. The 3-T MRI, which was used in the present study, provides a higher signal-to-noise ratio than 1.5-T MRI, resulting in improved spatial resolution, better image quality, and more accurate measurement.

Sundberg et al. [31] reported that 3.0-T MRI was as effective as 1.5-T d-MRA at detecting labral tears in all subjects. High-resolution T2*-weighted imaging, such as MERGE, is used to obtain detailed anatomical information on the cartilage or labrum in the hip and other joints [32]. Furthermore, spinal imaging with MERGE has shown high sensitivity for depicting edematous and hemorrhagic changes [33] as well as excellent depiction of soft tissue structures such as intervertebral discs and the ligamentum flavum in degenerative diseases of the cervical spine [34]. In the present study, we decided to use 3D-MERGE to focus on edema and degeneration of the labrum and to compare the results with the pathological degeneration grade.

Before starting this study, we had resected labra from 14 patients with osteoarthritis during THA and inspected the radial sections macroscopically. All patients had given informed consent. The radial sections showed a variety of shapes including triangular, square, polygonal, oval, and irregular, such as L- or V-shaped. The majority of specimens could not be classified into any of the three typical categories (i.e., triangular, round, or flat) for normal labra [17]. For classification of labral shape in advanced osteoarthritis, more than eight categories appear to be necessary. Since classification into many categories may not be readily reproducible, we used the simple classification of triangular and non-triangular. For labral tears, we evaluated only the major detachment from the acetabular rim during surgery. Evaluation of minor tears at the acetabular attachment of the labrum was difficult before resection and impossible in the resected specimen because the labrum was resected from the bony acetabular rim. Thus, we did not discuss labral tears here.

This study revealed that $81 \%$ of the MRI slices were concordant with macroscopic findings for all evaluations, including the presence, size, shape, and detachment of the labrum. The macroscopic findings of the ten unidentified MRI slices showed that the labrum was absent in six specimens and present in four. The discordance for the presence of the labrum in four specimens may have been caused by the formation of bony spurs at the femoral head and acetabulum, infiltration by surrounding synovial fluid, or degenerative changes of the labrum. On the other hand, labral detachment was accurately detected on non-contrast MRI in our protocol, and a substantial correlation was observed between SIR and histological grade (Spearman's rank correlation coefficient, 0.568 ). These results suggest that 3-T MRI can depict labral degeneration as changes in signal intensity.

There are several limitations inherent in our study design. Methods for measuring signal intensity by calculating the signal intensity ratio (SIR) remain unpopular in hip joint imaging, though it is widely accepted in other diagnostic imaging 
fields [13-15]. Regarding this point, a consensus reached by two readers might be suboptimal for these measuring methods. However, the intra- and interobserver reliability analysis of SIR showed good consistency, and we think these methods are applicable in hip joint imaging too. We did not compare the present method in terms of other acquisition parameters. In this SIR method, the signal intensity of the labrum was divided by the signal intensity of the rectus femoris tendon as the reference tissue in our study. The labrum-to-tendon signal ratio depends on acquisition parameters. Further studies using other pulse sequences should be performed. In the future, standardizing the parameter and the reference tissue would be optimal. The microscopic assessment of the degenerative area in each labrum was not objective, even though the inter- and intraobserver reliabilities were both high. The study was performed in elderly patients (mean age, 67 years), and at present, candidates for preoperative MRI evaluations are typically younger patients under consideration for jointpreserving procedures. Although our specimens included labra with mild degenerative changes, the possible effects of patient age should be taken into consideration. The correlations between signal intensity and the different types of degenerative conditions such as myxoid degeneration, fibrillation, neovascularization, cystic change, and hyaline cartilaginous change were not evaluated. Therefore, a comparative study with objective assessment of microscopic features is necessary to clarify the effectiveness of the presented MRI protocol. The assessment of the grade of microscopic degeneration was based only on HE staining. Further studies including qualitative assessment for proteoglycan are needed for better understanding of the correlation between MRI and microscopic findings.

In conclusion, a substantial correlation was observed between the signal intensity seen on 3-T MRI and the degeneration grade of the labrum seen histologically in osteoarthritic hips.

Acknowledgments We thank Dr. Tetsuo Imamura for providing advice on the pathology evaluation, Mr. Isao Yokota for providing advice on statistical analysis, and Ms. Yuko Hotta for assisting with specimen preparation.

Conflict of interest No benefits in any form have been received or will be received from a commercial party related directly or indirectly to the subject of this article.

\section{References}

1. Toomayan GA, Holman WR, Major NM, Kozlowicz SM, Vail TP. Sensitivity of MR arthrography in the evaluation of acetabular labral tears. AJR Am J Roentgenol. 2006;186(2):449-53.

2. Bittersohl B, Miese FR, Hosalkar HS, et al. T2* mapping of hip joint cartilage in various histological grades of degeneration. Osteoarthr Cartil. 2012;20(7):653-60.
3. Watanabe A, Boesch C, Siebenrock K, Obata T, Anderson SE, et al. T2 mapping of hip articular cartilage in healthy volunteers at 3T: a study of topographic variation. J Magn Reson Imaging. 2007;26(1): 165-71.

4. Keenan KE, Besier TF, Pauly JM, et al. Prediction of glycosaminoglycan content in human cartilage by age, T1p and T2 MRI. Osteoarthr Cartil. 2011;19(2):171-9.

5. Domayer SE, Mamisch TC, Kress I, Chan J, Kim YJ. Radial dGEMRIC in developmental dysplasia of the hip and in femoroacetabular impingement: preliminary results. Osteoarthr Cartil. 2010;18(11):1421-8.

6. Gold SL, Burge AJ, Potter HG. MRI of hip cartilage: joint morphology, structure, and composition. Clin Orthop Relat Res. 2012;470: 3321-31.

7. Roemer FW, Hunter DJ, Winterstein A, et al. Hip osteoarthritis MRI scoring system (HOAMS): reliability and associations with radiographic and clinical findings. Osteoarthr Cartil. 2011;19(8):946-62.

8. Kubo T, Horii M, Yamaguchi J, et al. Radial magnetic resonance imaging and pathological findings of acetabular labrum in dysplastic hips. Pathophysiology. 2000;7(3):171-5.

9. Altman R, Alarcón G, Appelrouth D, et al. The American College of Rheumatology criteria for the classification and reporting of osteoarthritis of the hip. Arthritis Rheum. 1991;34(5):505-14.

10. Arnett FC, Edworthy SM, Bloch DA, et al. The American Rheumatism Association 1987 revised criteria for the classification of rheumatoid arthritis. Arthritis Rheum. 1988;31(3):315-24.

11. Sugano N, Kubo T, Takaoka K, et al. Diagnostic criteria for nontraumatic osteonecrosis of the femoral head. A multicentre study. J Bone Joint Surg (Br). 1999;81(4):590-5.

12. Tönnis D, Heinecke A. Acetabular and femoral anteversion: relationship with osteoarthritis of the hip. J Bone Joint Surg Am. 1999;81(12):1747-70.

13. Yamamoto A, Oba H, Furui S. Influence of age and sex on signal intensities of the posterior lobe of the pituitary gland on T1-weighted images from 3T MRI. Jpn J Radiol. 2013;31(3):186-91.

14. Kanda T, Ishii K, Kawaguchi H, Kitajima K, Takenaka D. High signal intensity in the dentate nucleus and globus pallidus on unenhanced T1-weighted MR images: relationship with increasing cumulative dose of a gadolinium-based contrast material. Radiology. 2014;270(3):834-41.

15. Hernando D, Levin YS, Sirlin CB, Reeder SB. Quantification of liver iron with MRI: state of the art and remaining challenges. J Magn Reson Imaging. 2014;40(5):1003-21.

16. Cotton A, Boutry N, Demondion X, et al. Acetabular labrum: MRI in asymptomatic volunteers. J Comput Assist Tomogr. 1998;22(1):1-7.

17. Aydingöz U, Oztürk MH. MR imaging of the acetabular labrum: a comparative study of both hips in 180 asymptomatic volunteers. Eur Radiol. 2001;11(4):567-74.

18. Hodler J, Yu JS, Goodwin D, Haghighi P, Trudell D, Resnick D. MR arthrography of the hip: improved imaging of the acetabular labrum with histologic correlation in cadavers. AJR Am J Roentgenol. 1995;165(4):887-91.

19. Philippon MJ, Briggs KK, Yen YM, Kuppersmith DA. Outcomes following hip arthroscopy for femoroacetabular impingement with associated chondrolabral dysfunction: minimum two-year followup. J Bone Joint Surg (Br). 2009;91(1):16-23.

20. Schilders E, Dimitrakopoulou A, Bismil Q, Marchant P, Cooke C. Arthroscopic treatment of labral tears in femoroacetabular impingement:a comparative study of refixation and resection with a minimum two-year follow-up. J Bone Joint Surg (Br). 2011;93(8):1027-32.

21. Rakhra KS. Magnetic resonance imaging of acetabular labral tears. J Bone Joint Surg Am. 2011;93(2):28-34.

22. Mintz DN, Hooper T, Connell D, Buly R, Padgett DE, Potter HG. Magnetic resonance imaging of the hip: detection of labral and chondral abnormalities using noncontrast imaging. Arthroscopy. 2005;21(4):385-93. 
23. James S, Miocevic M, Malara F, Pike J, Young D, Connell D. MR imaging findings of acetabular dysplasia in adults. Skelet Radiol. 2006;35(6):378-84.

24. Yasunaga Y, Ochi M, Terayama H, Tanaka R, Yamasaki T, Ishii Y. Rotational acetabular osteotomy for advanced osteoarthritis secondary to dysplasia of the hip. J Bone Joint Surg Am. 2006;88(9):1915-9.

25. Hasegawa Y, Kanoh T, Seki T, Matsuoka A, Kawabe K. Joint space wider than $2 \mathrm{~mm}$ is essential for an eccentric rotational acetabular osteotomy for adult hip dysplasia. J Orthop Sci. 2010;15(5):620-5.

26. Nakamura S, Ninomiya S, Takatori Y, Morimoto S, Umeyama T. Long-term outcome of rotational acetabular osteotomy: 145 hips followed for 10-23 years. Acta Orthop Scand. 1998;69(3):259-65.

27. Cunningham T, Jessel R, Zurakowski D, Millis MB, Kim YJ. Delayed gadolinium-enhanced magnetic resonance imaging of cartilage to predict early failure of Bernese periacetabular osteotomy for hip dysplasia. J Bone Joint Surg Am. 2006;88(7):1540-8.

28. Yasunaga Y, Yamasaki T, Ochi M. Patient selection criteria for periacetabular osteotomy or rotational acetabular osteteotomy. Clin Orthop Relat Res. 2012;470(12):3342-54.
29. Kim KI, Cho YJ, Ramteke AA, Yoo MC. Peri-acetabular rotational osteotomy with concomitant hip arthroscopy for treatment of hip dysplasia. J Bone Joint Surg (Br). 2011;93(6):732-7.

30. Ueshima K, Takahashi KA, Fujioka M, et al. Relationship between acetabular labrum evaluation by using radial magnetic resonance imaging and progressive joint space narrowing in mild hip dysplasia. Magn Reson Imaging. 2006;24(5):645-50.

31. Sundberg TP, Toomayan GA, Major NM. Evaluation of the acetabular labrum at 3.0-T MR imaging compared with 1.5-T MR arthrography: preliminary experience. Radiology. 2006;238(2): 706-11

32. Theysohn JM, Kraff O, Theysohn N, et al. Hip imaging of avascular necrosis at 7 Tesla compared with 3 Tesla. Skelet Radiol. 2014;43(5): 623-32.

33. Martin N, Malfair D, Zhao Y, et al. Comparison of MERGE and axial T2-weighted fast spin-echo sequences for detection of multiple sclerosis lesions in the cervical spinal cord. AJR Am J Roentogenol. 2012;199(1):157-62.

34. Vertinsky AT, Krasnokutsky MV, Augustin M, Bammer R. Cuttingedge imaging of the spine. Neuroimaging Clin N Am. 2007;17(1): $117-36$. 